\title{
São Paulo. Entwicklungstrends einer brasilianischen Megastadt
}

Martin Coy, Tübingen

\section{Die Rahmenbedingungen: Verstädterung in Brasilien}

In nur wenigen Jahrzehnten hat sich Brasilien von einem vorwiegend ruralen in ein weitgehend verstädtertes Land verwandelt. Während um 1940 noch $70 \%$ der Bevölkerung auf dem Lande lebte, ist dieser Anteil bis zur Jahrtausendwende auf unter $20 \%$ zurückgegangen. Besonders eindrucksvoll ist das Stadtwachstum in absoluten Zahlen: 1940 lebten erst ca. 13 Millionen Menschen in den Städten, im Zensusjahr 1996 waren es bereits über 120 Millionen. Allein in den 80er Jahren, der Phase ihrer stärksten Zunahme, sind die brasilianischen Städte um über 30 Millionen Menschen gewachsen (vgl. Abbildung 1b). Auch wenn das Land bereits einen relativ hohen Verstädterungsgrad von knapp über $80 \%$ aufweist, geht der Urbanisierungsprozeß unvermindert weiter. Dabei zeigt die Verteilung der städtischen Bevölkerung auf die fünf Großregionen des Landes (vgl. Abbildung 1b) nach wie vor das eindeutige Übergewicht des Südostens, der bis in die 80er Jahre ca. $50 \%$ der in Städten lebenden Brasilianer beherbergte. Erst mit Abstand folgen der Nordosten und der Süden. Bezogen auf Gesamtbrasilien fällt demgegenüber die Stadtbevölkerung in den Großregionen Norden und Mittelwesten kaum ins Gewicht. Allerdings ist in den letzten 30 Jahren der Anteil dieser beiden Regionen an der Gesamt-Stadtbevölkerung Brasiliens aufgrund besonders dynamischer Regionalentwicklungsprozesse und des damit zusammenhängenden Wachstums der Mittelstädte stark angestiegen (vgl. als Fallbeispiel Cor 1999).

Über viele Jahrzehnte hinweg prägte jedoch vor allem die Metropolisierung den brasilianischen Verstädterungsprozeß. So stellen die Metropolitanregionen von São Paulo und Rio de Janeiro heute mit weitem Abstand die größten urbanen Agglomerationen des Landes dar und zählen gleichzeitig zu den größten Megastädten der Erde (vgl. Abbildung 1a). Für das Zensusjahr 1996 werden die Einwohnerzahlen der Metropolitanregion São Paulo mit knapp 17 Millionen und die Rio de Janeiros mit etwas über 10 Millionen angegeben. Die übrigen sieben Metropolitanregionen Brasiliens folgen mit erheblichem Abstand. Allerdings ist die Wachstumsdynamik der Metropolitanregionen sehr unterschiedlich (vgl. Abbildung 1a): Während der 90er Jahre (1991 bis 1997) wuchs Rio de Janeiro nur noch um ca. 5\%, gefolgt von Recife (7\%), Porto Alegre und São Paulo (jeweils 9\%). Das relativ stärkste Wachstum verzeichnete im genannten Zeitraum Curitiba mit ca. 22\% (Daten nach offiziellen Angaben des IBGE, Instituto Brasileiro de Geografia e Estadística). Dabei ist in allen Metropolitanregionen die Dynamik des Bevölkerungswachstums in den Gemeinden der Peripherie, in denen sich in den meisten Fällen hohe Anteile armer Bevölkerung finden, inzwischen deutlich höher als in den Kernstädten.

Neben den quantitativen Aspekten des brasilianischen Verstädterungsprozesses sind insbesondere seine qualitativen, innerstädtischen Implikationen von Bedeutung (vgl. Abbildung 2). Stadtwachstum beinhaltet sowohl Zuwachs der Bevölkerung als auch eine unkontrollierte Flächenexpansion. Infolge dieses unkontrollierten Wachstumsprozesses verschärfen sich insbesondere die Fragmentierung der städtischen Gesellschaft sowie die innerstädtische sozialräumliche Segregation (vgl. zu den räumlichen Prozessen in lateinamerikanischen Großstädten insgesamt BäHR \& MERTINs 1995). So sind die Städte einerseits die Orte, in denen sich die Reichen und Erfolgreichen mit Hochhausquartieren, abgeschlossenen Wohnsiedlungen und Shopping Center oftmals auf Kosten der Innenstadtbereiche ihre eigenen Aktions- und Repräsentationsräume schaffen. Auf der anderen Seite werden immer größere Areale an den Stadträndern oder auf marginalen Flächen zu Überlebensräumen der Armen. Ein Großteil der Bewohner brasilianischer Städte lebt heute in Marginalvierteln, oftmals Landbesetzungen, in denen es entweder überhaupt keine oder allenfalls höchst prekäre Infrastrukturen gibt. Arbeit und ein geringes Einkommen bietet häufig nur der informelle Sektor. Ein Großteil der städtischen Bevölkerung ist dadurch nicht in der Lage, die elementarsten Grundbedürfnisse zu befriedigen. So spiegeln sich heute in den Städten die Widersprüche der brasilianischen Gesellschaft in besonderem Maße wieder.

Aktueller Strukturwandel und Entwicklungsperspektiven insbesondere der brasilianischen Metropolitanräume stehen zunehmend unter dem Vorzeichen der Globalisierung. In wirtschaftlicher Hinsicht sind die städtische Industrie, die Finanzmärkte und der Kommunikationssektor immer enger mit dem globalen System verflochten (vgl. SASSEN 1996: 50 ff.). Vor allem São Paulo ist bereits in die unterschiedlichen «Listen» der global cities als übergeordnete Steuerungszentrale von großregionaler Bedeutung integriert (vgl. z.B. FRIEDMANN 1995: 25). Unter soziokulturellem Blickwinkel wirkt sich die Globalisierung der Lebensstile in der Homogenisierung der gebauten Umwelt in der Stadt sowie in Veränderungen städtischer Konsum- 

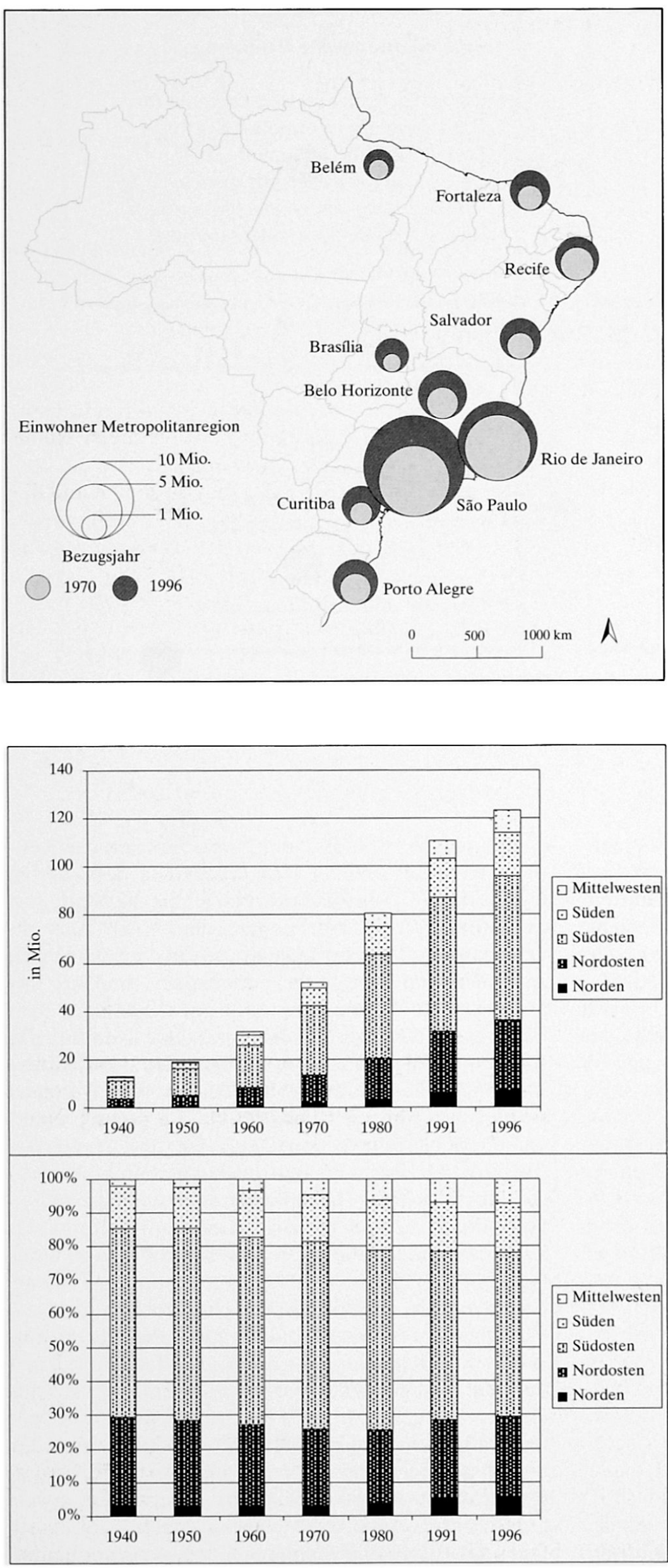

Abb. 1a: Bevölkerungsentwicklung der Metropolitanregionen in Brasilien 1970 1996

Population development in metropolitan regions of Brazil 1970-1996

L'évolution démographique des régions métropolitaines au Brésil (1970-1996)

Quelle: Karten- und Datengrundlage: IBGE 2001; Entwurf: M. Coy, Zeichnung: M. PÖHLER
Abb. 1b: Entwicklung der städtischen Bevölkerung nach Grossregionen in Brasilien 1940 - 1996

Urban population development in Brazil 1940-1996, according to regions

L'évolution de la population urbaine au Brésil par grandes régions (1940-1996)

Quelle: Karten- und Datengrundlage: IBGE 2001; Entwurf: M. Cor, Zeichnung: M. PÖHLER 


Stadtwachstum
- Bevölkerungswachstum:
- Zuwanderung
- Natürliches Wachstum

Abb. 2: Städtische Probleme in Brasilien heute

M. Coy 2000 Urban problems in Brazil today Problèmes urbains au Brésil d'aujourd'hui

muster und Wohnformen aus (siehe hierzu beispielsweise Frúgolı JR. 1995). Insgesamt verstärken Privatisierung, Deregulierung und Flexibilisierung nicht nur den wirtschaftlichen Wandel, sondern auch die sozialen Disparitäten, so daß die zunehmende Fragmentierung der Städte zwischen Zitadellenbildung und Ghettoisierung als grundlegendes Charakteristikum der brasilianischen Stadtentwicklung der letzten Jahre unter den Vorzeichen der Globalisierung anzusehen ist (vgl. für die Entwicklungsländer generell ScHOLz 2000).

Vor diesem Hintergrund analysiert der Beitrag am Beispiel von São Paulo Ursachen und Ausprägungen des aktuellen innerstädtischen Wandels und der damit einhergehenden funktional- und sozialräumlichen Fragmentierung. Anschließend werden die Bemühungen von Stadtverwaltung und Nicht-Regierungsorganisationen um eine Revitalisierung des Stadtzentrums beispielhaft für die stadtpolitischen Akteurskonstellationen und Handlungsspielräume aufgezeigt.

\section{Entwicklungstendenzen einer Megalopolis: Das Beispiel São Paulo}

Der Aufstieg São Paulos zur inzwischen - vor Mexico City - größten Megastadt Lateinamerikas und zum wichtigsten Wirtschafts- und Finanzzentrum des Kontinents vollzog sich im Wesentlichen in der zweiten Hälfte des 20. Jahrhunderts. Nachdem die Stadt erst gegen Ende des 19. Jahrhunderts die 100.000-Einwohner-Schwelle überschritten hatte und in den 30 er Jahren zur Millionen-Metropole heranwuchs, brachte vor allem die im Rahmen des assoziativ-kapitalistischen Modells der 50er bis 70er Jahre auf der Förderung der Industrie basierende Entwicklung den entscheidenden Wachstumsschub (vgl. KoHLHEPP 1997). Während sich im erweiterten Zentrum der Kernstadt zunehmend die Standorte von Zentralen und Niederlassungen zahlreicher in- und ausländischer Unternehmen des Industrie-, Dienstleistungs- und Finanzsektors konzentrierten, bildeten sich in den an die Kernstadt angrenzenden Kommunen des Metropolitanraumes (vgl. Abb. 3) große industrielle Standortagglomerationen heraus. Beispielsweise entwickelten sich die Gemeinden des sogenannten ABC Paulista (Santo André, São Bernardo do Campo und São Caetano do Sul) mit den Produktionsstätten zahlreicher ausländischer Konzerne (z.B. VW) zu einem der bedeutendsten Standorte des Automobilsektors und der metallverarbeitenden Industrie nicht nur des Landes, sondern auch des gesamten Subkontinents. Im erweiterten Umland befindet sich in Cubatão auf der Basis der Eisen- und Stahlerzeugung, der chemischen, 


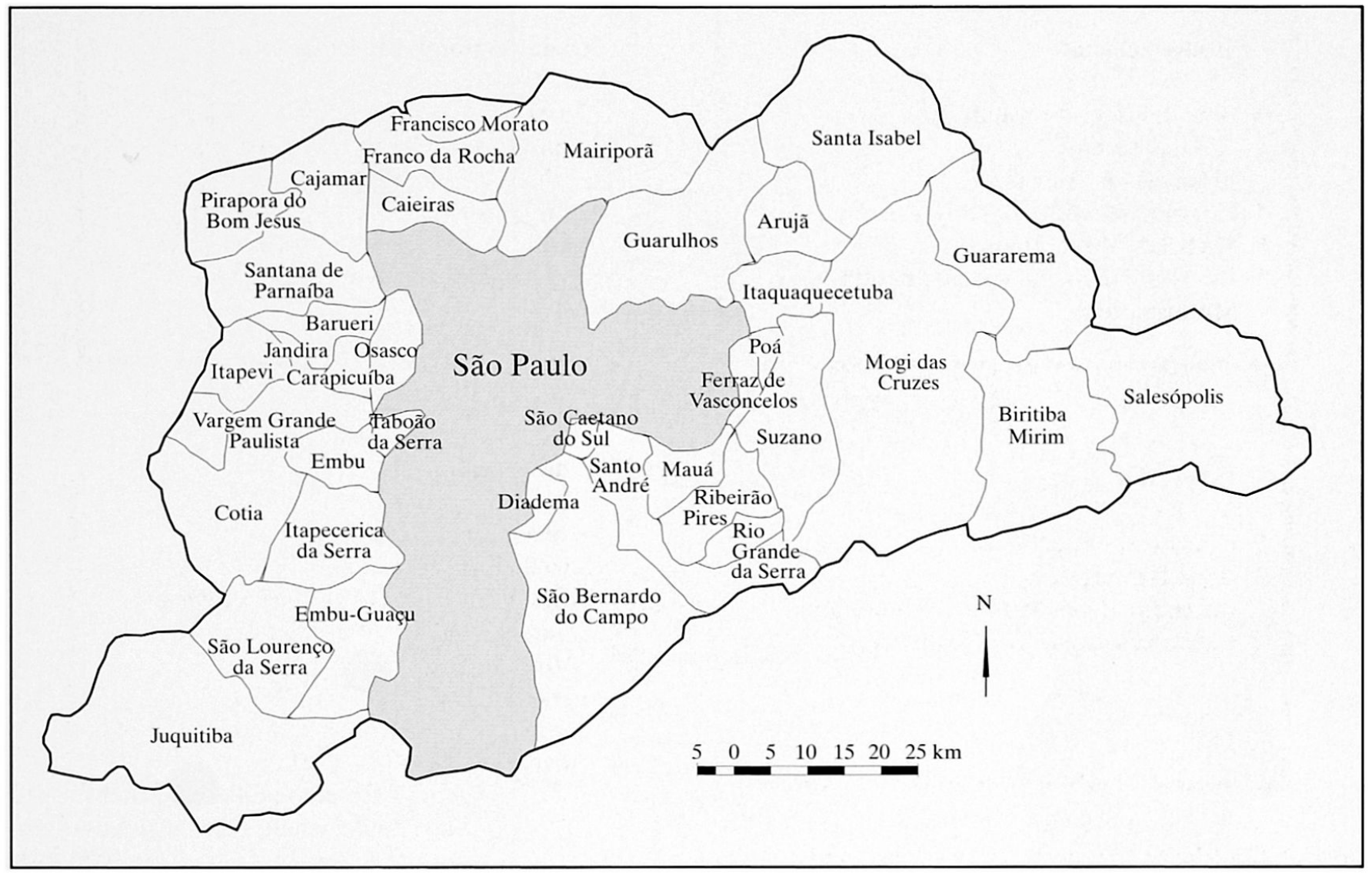

Abb. 3: Die Metropolitanregion São Paulo

The Metropolitan Area of São Paulo

La région métropolitaine de São Paulo

Quelle: Kartengrundlage: EMPLASA; Entwurf: M. CoY, Zeichnung: M. PöHLER

petrochemischen und pharmazeutischen Industrie einer der größten Industriestandorte Lateinamerikas, der gleichzeitig unter schwerwiegenden sozialen und ökologischen Folgeproblemen zu leiden hat (vgl. GutBERLET 1991). Die Präsenz ausländischen Kapitals prägt den Wirtschaftsstandort São Paulo in besonderer Weise. So gilt die Region beispielsweise als weltweit größte Agglomeration deutscher Firmen außerhalb Deutschlands.

Die dynamische Wirtschaftsentwicklung im Großraum São Paulo ist der entscheidende Grund für die große Attraktivität, die die Metropole auf Zuwanderer aus allen Landesteilen, insbesondere aus dem brasilianischen Nordosten, seit Mitte des 20. Jahrhunderts ausübt. Zwischen 1960 und 2000 hat sich die Bevölkerung der Metropolitanregion von knapp 5 Millionen auf fast 18 Millionen mehr als verdreifacht. Dabei fand das rascheste Wachstum in den 60er und 70er Jahren - der Hochphase des brasilianischen "Wirtschaftswunders» - statt, um sich dann ab den $80 \mathrm{er}$ Jahren wieder zu verlangsamen. Ergebnis dieses außerordentlichen Wachstumsprozesses ist eine hin- sichtlich ihrer sozioökonomischen und räumlichen Entwicklungsdynamik sowie hinsichtlich ihrer Strukturen und alltagsweltlichen Lebensbedingungen zutiefst fragmentierte Metropolitanregion (vgl. Santos 1990, VILLAÇA 1998).

Vom Bevölkerungswachstum waren die außerhalb der Kernstadt gelegenen Munizipien des Metropolitanraumes überproportional betroffen. Während 1960 die Kernstadt noch $77 \%$ der Bevölkerung der Gesamtagglomeration stellte, ging bis 2000 dieser Anteil auf $59 \%$ zurück (das entspricht knapp 10 Millionen Einwohnern). Besonders beeindruckend ist das Wachstum der östlich der Kernstadt gelegenen Gemeinde Guarulhos, deren Bevölkerung sich seit 1960 verzehnfachte und die inzwischen die zweitgrößte Stadt des Metropolitanraumes ist. In diesem Teilraum ist der Anteil ärmerer Bevölkerung besonders hoch.

Ganz anders als in den durchweg wachsenden suburbanen Munizipien sieht die Bevölkerungsentwicklung in São Paulo selbst aus. Zwar hat sich auch die Einwohnerzahl der Kernstadt von 1960 bis 2000 fast ver- 


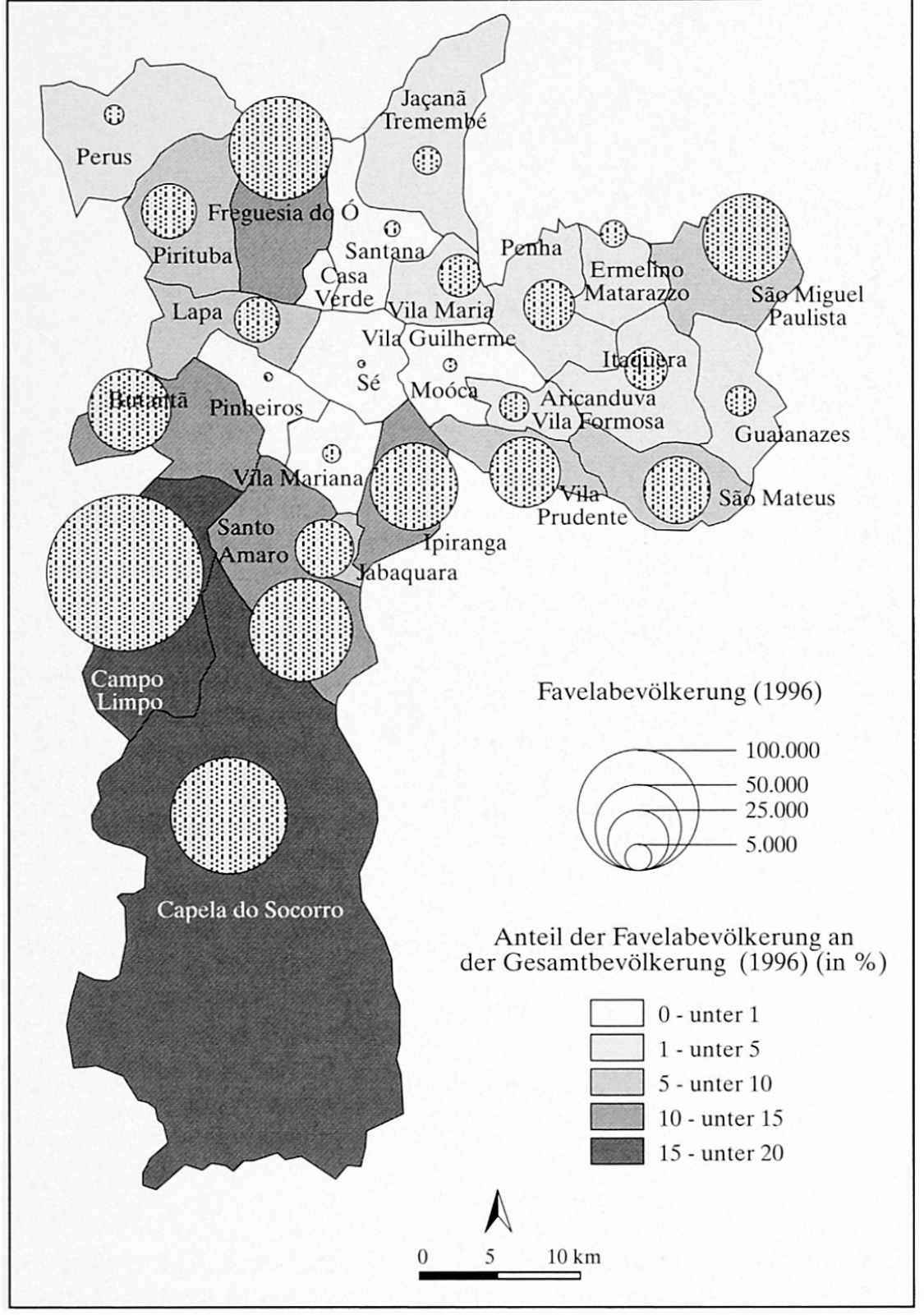

Abb. 4:Favelabevölkerung im Munizip São Paulo nach Administrativregionen 1996

Population living in "favelas" within the «Munizip» of São Paulo, according to administrative regions 1996

La population des favelles dans le municipe de São Paulo, par régions administratives (1996)

Quelle: Karten- und Datengrundlage: EMPLASA (Empresa Metropolitana de Planejamento da Grande São Paulo S.A.) 2001; Entwurf: M. Coy, Zeichnung: M. PÖHLER dreifacht, jedoch sind innerhalb der Stadt deutliche Zentrum-Peripherie-Unterschiede festzustellen. Insbesondere die innenstadtnahen Viertel haben deutliche Bevölkerungsverluste hinnehmen müssen. So hat sich beispielsweise die Wohnbevölkerung im eigentlichen Zentrum im relativ kurzen Zeitraum von 1980 bis 2000 fast halbiert. Ebenso sind die Einwohnerzahlen sowohl in den bessergestellten zentrumsnahen Quartieren im Westen als auch in den einfacheren Quartieren im Osten des Zentrums deutlich zurückgegangen. Zuwächse verzeichneten dagegen die zentrumsferneren Viertel im Nordosten, Osten und Südwesten.

Die gegenläufigen Trends der Bevölkerungsentwicklung zwischen innerstädtischen und peripheren Quartieren hängen im Wesentlichen mit zwei Ursachenkomplexen zusammen: Hohe Baudichte, Konzentration von Einzelhandels- und Bürofunktion sowie damit ein- hergehende extreme Verkehrsbelastung im engeren und erweiterten Zentrum sind mit der Verdrängung der Wohnfunktion verbunden (siehe zur Zentrumsentwicklung auch weiter unten). Wohnraum wird in Büroraum verwandelt oder durch diesen ersetzt, und diejenigen, die es sich leisten können, ziehen - auch vor dem Hintergrund der gestiegenen individuellen Mobilität - in zentrumsfernere Gebiete mit höherer Wohnumfeldqualität. Hiervon profitieren die randlichen, weniger dicht bebauten Areale. In diesen Randgebieten dehnen sich - teilweise in räumlicher Nähe zu bessergestellten Wohnvierteln - jedoch vor allem die mit der zumeist illegalen Besetzung von öffentlichem und privatem Land enstehenden Marginalviertel (Favelas) aus (vgl. Abb. 4 und Foto 1).

Je nach Quelle schwanken - unter anderem aus methodischen Erhebungsgründen - die Angaben zum in 


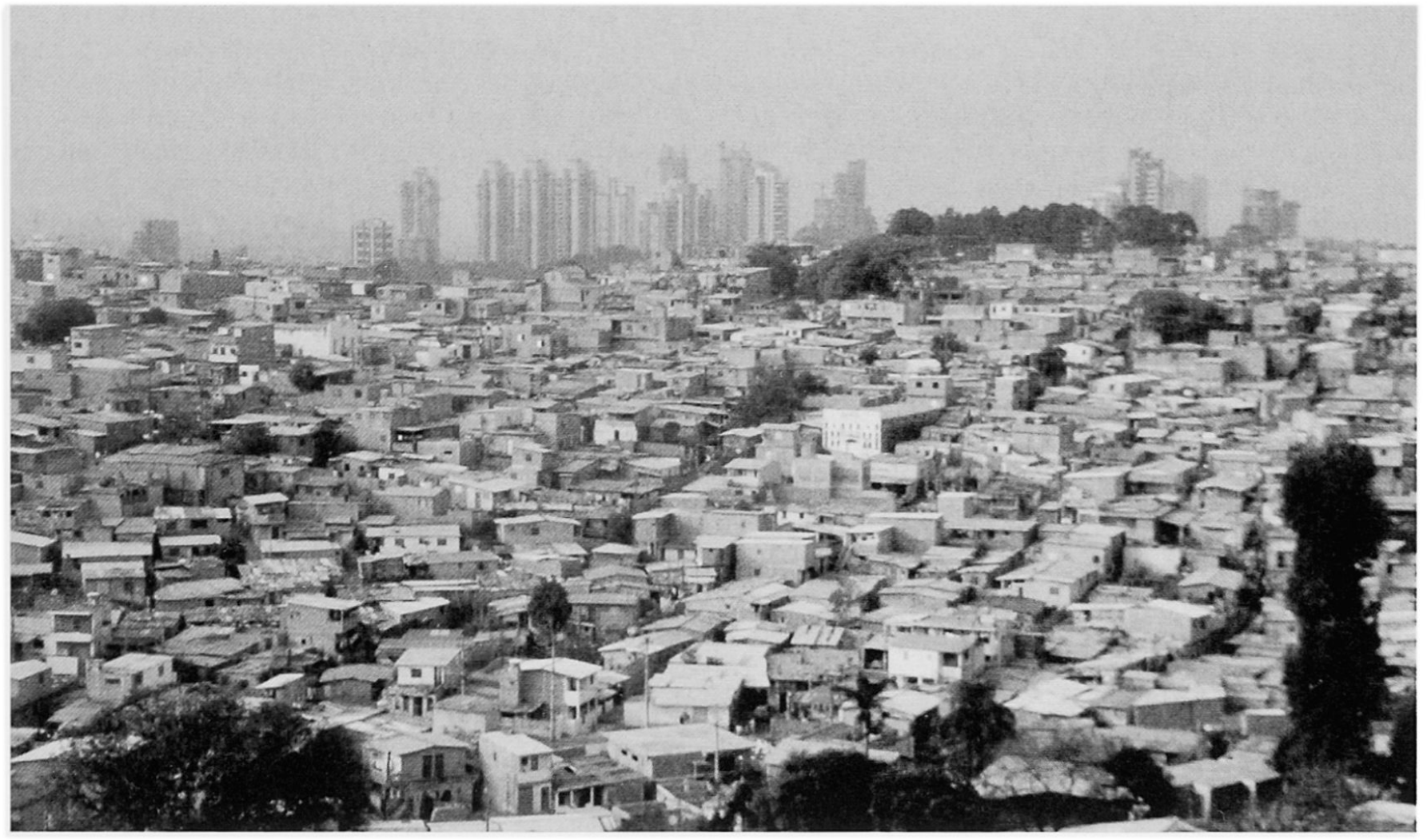

Foto 1: Sozialräumliche Segregation in São Paulo: eine Favela in Nachbarschaft zu Oberschichtvierteln im Stadtteil Morumbi

Socio-spatial segregation in São Paulo: a "favela» neighbouring an upper-class area in the urban district of Morumbi

Ségrégation socio-spatiale à São Paulo: une favelle à proximité des quartiers habités par les couches supérieures de la population à Morumbi

Foto: F. ZIRKL 1999

Favelas lebenden Bevölkerungsanteil von São Paulo erheblich (vgl. zur Genese der Favelas von São Paulo BoNDUKı 1998: $264 \mathrm{ff}$.). Als gesichert gilt, daß die in Favelas lebende Bevölkerung seit 1970 im Verhältnis zur Gesamtbevölkerung der Stadt überproportional angewachsen ist. So werden für 1970 lediglich $1 \%$, für 1987 bereits $8 \%$ genannt. Jüngere wissenschaftliche Untersuchungen haben für 1993 ergeben, daß fast $20 \%$ der Bevölkerung São Paulos (das entsprach damals mehr als 1,5 Millionen) in Favelas lebten (vgl. Maricato 1996: 27). Deutlich tritt in Abbildung 4 die Konzentration der Favelas in den Quartieren der südlichen, der östlichen und teilweise der nördlichen Peripherie der Stadt zutage. Einer Erhebung der städtischen Wohnungsbaubehörde aus dem Jahr 1987 zufolge waren von den zum damaligen Zeitpunkt existierenden ca. 1.600 Favelas der Stadt 65\% durch Besetzung öffentlichen Landes entstanden. Ca. 50\% dieser Favelas lagen zumindest teilweise in Uferrandbereichen von Flüssen und Bächen und waren deshalb von Überschwemmungsgefahr besonders bedroht. $30 \%$ befanden sich an Hängen, was bei den saisonal auftretenden Starkregenereignissen mit besonderen Rutschungsgefährdungen verbunden ist. $1 \%$ der damals untersuchten Favelas waren auf Müllkippen angelegt (vgl. zu den Angaben Maricato 1996: 58). Zusätzlich zu der völlig unzureichenden Versorgung der Marginalviertel mit sozialen und hygienischen Basisinfrastrukturen weisen diese Angaben zur rechtlichen Situation und räumlichen Lage auf die besondere Verwundbarkeit der Armenviertel hin, die sich entsprechend in jährlich auftretenden Katastrophen mit zahlreichen Todesfällen ausdrücken.

Auch wenn die Zahl der randstädtischen Marginalviertel in den letzten Jahren besonders stark zugenommen hat, so sind die Wohnquartiere der Armen aus der Innenstadt keineswegs vollständig verschwunden. Im Gegenteil: seit ca. 100 Jahren existiert insbesondere in zentrumsnahen Vierteln São Paulos das Phänomen der Cortiços, Behausungen mit zumeist subhumanen Wohnbedingungen, erheblicher Überbelegung, ungeklärter Miet- bzw. Eigentumssituation, die sich oftmals in den ehemaligen Wohngebieten der Bessergestellten 
bilden (vgl. Kowarick \& ANT 1994, BonduKI 1998). Nach wissenschaftlichen Untersuchungen von FIPE/ USP (Fundação Instituto de Pesquira Econômica/ Universidade de São Paulo) aus der Mitte der 90er Jahre sollen über 600.000 Menschen in den Cortiços von São Paulo leben. Insofern stellt diese Wohnform eine nach wie vor wichtige Überlebensstrategie der Unterschichtbevölkerung in der Megalopolis dar, zumal sie gegenüber den randstädtischen Favelas ihren zumeist im informellen Sektor tätigen Bewohnern den Vorteil der Nähe zum Zentrum als «Arbeitsplatz» bieten. Auch die am untersten Ende der sozialen Rangskala lebenden Stadtbewohner, die sogenannte População da Rua, die Obdachlosen und Straßenkinder, sind vor allem im Zentrumsbereich der Stadt zu finden. Nach Angaben der Stadtverwaltung für das Jahr 2000 halten sich von den offiziell erfaßten ca. 9.000 permanent Obdachlosen (die realen Zahlen dürften weitaus höher liegen) drei Viertel in den verschiedenen Quartieren des erweiterten Zentrums auf.

\subsection{Das Stadtzentrum von São Paulo im Wandel}

Die Häufung von Cortiços und die Konzentration der População de Rua können als Indikatoren für den tiefgreifenden sozialräumlichen Wandel im Stadtzentrum von São Paulo angesehen werden. Bis vor wenigen Jahrzehnten waren im unmittelbaren Stadtzentrum noch zahlreiche hochrangige Wohngebiete zu finden, und der öffentliche Raum des Zentrums, seine Cafés, Restaurants, Kinos und Theater dienten den Repräsentations- und Kommunikationsbedürfnissen der «besseren» Gesellschaft der aufstrebenden Metropole. Der eigentliche Zentrumsbereich von São Paulo läßt sich in zwei durch das Vale do Anhangabaú unterteilte Bereiche gliedern: das sogenannte alte Zentrum (Centro Velho) und das neue Zentrum (Centro Novo) (siehe Abb. 6 und Foto 2). Besonders das Centro Velho hat sich - neben den traditionellen Straßen des Einzelhandels - seit der ersten Hälfte des 20. Jahrhunderts mit Hauptsitzen und Niederlassungen in- und ausländischer Banken sowie den Börsen zum Zentrum des Finanzsektors entwickelt. Im Centro Novo sind neben den zahlreichen Büros insbesondere der Einzelhandel, Hotels und kulturelle Einrichtungen (Teatro Municipal, Kinos etc.) lokalisiert. Viele der hier zu findenden breiten Boulevards galten - und gelten teilweise heute noch - als hochwertige Wohnadressen. Charakteristisch für beide Zentrumsbereiche sind die bauliche Dichte und der hohe Vertikalisierungsgrad, der mit dem Bau der ersten Hochhäuser nach nordamerikanischem Vorbild bereits in den 20er Jahren einsetzte (vgl. Soмекн 1997, SouZa 1994). Nördlich an das Zentrum schließen sich die Areale der großen Bahnhöfe an, die für die überörtliche Anbindung der Stadt von Bedeutung waren. Ebenso befinden sich in Zentrumsnähe zahlreiche Wohnviertel, die durch die Vorherrschaft spezifischer Einwanderergruppen bereits früh die Kennzeichen ethnischer und sozialer Segregation aufwiesen und bis heute beibehalten haben (beispielsweise Brás und Bela Vista als Viertel der italienischstämmigen Bewohner, Liberdade als japanisch geprägtes Viertel).

Aufgrund der zunehmenden Verdichtung der Büround Einzelhandelsfunktionen im räumlich begrenzten Zentrumsbereich, der enormen Belastung durch den öffentlichen und den Individualverkehr, auf den das Zentrum durch den Ausbau von Verkehrsachsen und Hochstraßen ausgerichtet wurde, aufgrund der damit einhergehenden Verschlechterung des Wohnumfeldes sowie aufgrund der fehlenden Reserveflächen für den boomenden Tertiären Sektor verlagerten sich seit den 50er und 60er Jahren zahlreiche Zentrumsfunktionen

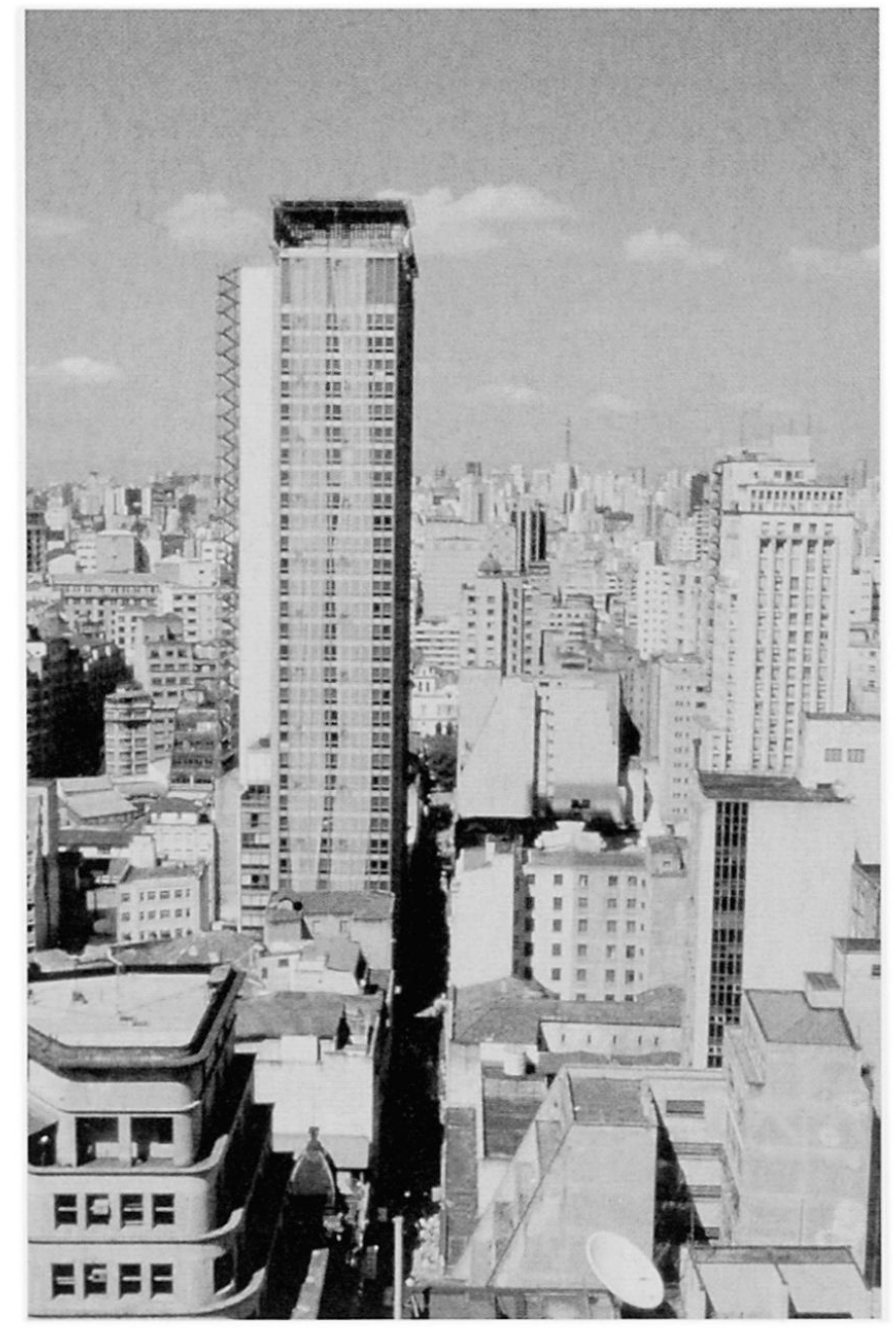

Foto 2: Bauliche Verdichtung im Stadtzentrum von São Paulo

Urban structural concentration in the city centre of São Paulo

Densité immobilière au centre de São Paulo

Foto: M. Cor 2000 
vor allem in westlich-südwestlicher Richtung. Hier entstanden beispielsweise mit den sogenannten Jardins hochwertige Wohngebiete. Die ehemals durch Prachtvillen der Kaffeebarone geprägte Avenida Paulista wurde zusehends zur Hochhausschlucht umgewandelt und erfüllt heute Funktionen einer zentralen Achse des Büro- und Finanzsektors. Der Verlagerungsprozeß des Dienstleistungsbereichs setzte sich auch in den 70er, 80 er und 90er Jahren in südwestlicher Richtung bis an die Grenzen der Kernstadt fort. In diesen Stadtgebieten entstehen zahlreiche mit allen modernen Kommunikationsinfrastrukturen ausgestattete Bürohochhäuser, und hier werden heute die höchsten Renditen des paulistaner Immobilienmarktes erzielt.

Die Verlagerung der bessergestellten Wohngebiete geht auch einher mit Veränderungen der Konsumge- wohnheiten und Konsumorte, die den Einzelhandel im «alten» Zentrum wesentlich beeinflußt haben. São Paulo nahm seit Mitte der 60er Jahre als erste brasilianische und lateinamerikanische Metropole das nordamerikanische Vorbild der Shopping Center auf, die sehr bald insbesondere in den südwestlichen Expansionsgebieten der Stadtentwicklung Funktionen der Zentrenbildung erfüllten (vgl. Abbildung 5). Als erstes innerstädtisches Einkaufszentrum entstand 1966 an der Av. Brig. Faria Lima das Shopping Center Iguatemi, das bis heute als eines der hochwertigsten der Stadt gilt. Zu Ende der 90er Jahre existierten allein in der Kernstadt São Paulo 50 Shopping Center, wobei die größten jeweils über 400 Geschäfte beherbergen. Insbesondere in den 80 er und 90er Jahren sind zahlreiche neue Shoppings auch in zentrumsferneren Gebieten entstanden (vgl. Abbildung 5). Neben den Zentren

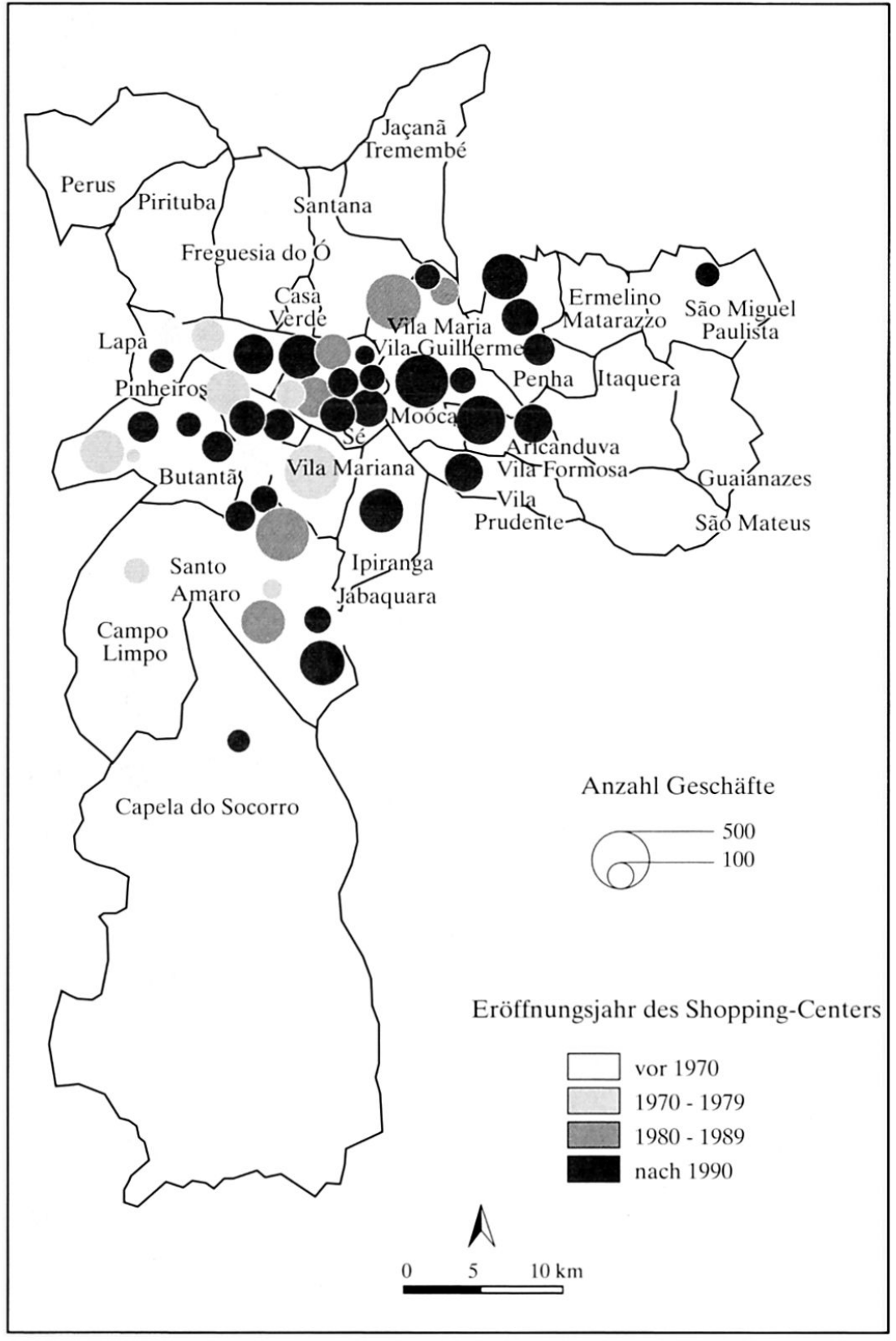

Abb. 5: Shopping-Center im Munizip São Paulo nach Administrativregionen 1998

Shopping-centres within the "Munizip» São Paulo according to administrative regions 1998

Les shopping-centers dans le municipe de São Paulo, par régions administratives (1998)

Quelle: Daten- und Kartengrundlage: EMPLASA 2001; Entwurf: M. CoY, Zeichnung: M. PÖHLER 
mit hochwertigem Geschäftsangebot sind dies nun auch zunehmend solche, die sich auf ein weniger kaufkräftiges Publikum in ihrem Einzugsbereich richten. Neben der reinen Geschäftsfunktion übernehmen die Shopping Center als privatkapitalistisch produzierte und kontrollierte Räume heutzutage zunehmend Kommunikations- und Freizeitfunktionen, die früher eher im Stadtzentrum oder im öffentlichen Raum lokalisiert waren (z.B. Kinos, Restaurants). Auch dies ist als Indiz einer zunehmenden Tendenz der sozialen Fragmentierung von städtischer Gesellschaft und städtischem Raum zu werten (vgl. FrúGOLI JR. 1995).

Neben den im Wohnbereich festzustellenden Degradierungsprozessen und den Auslagerungstendenzen im

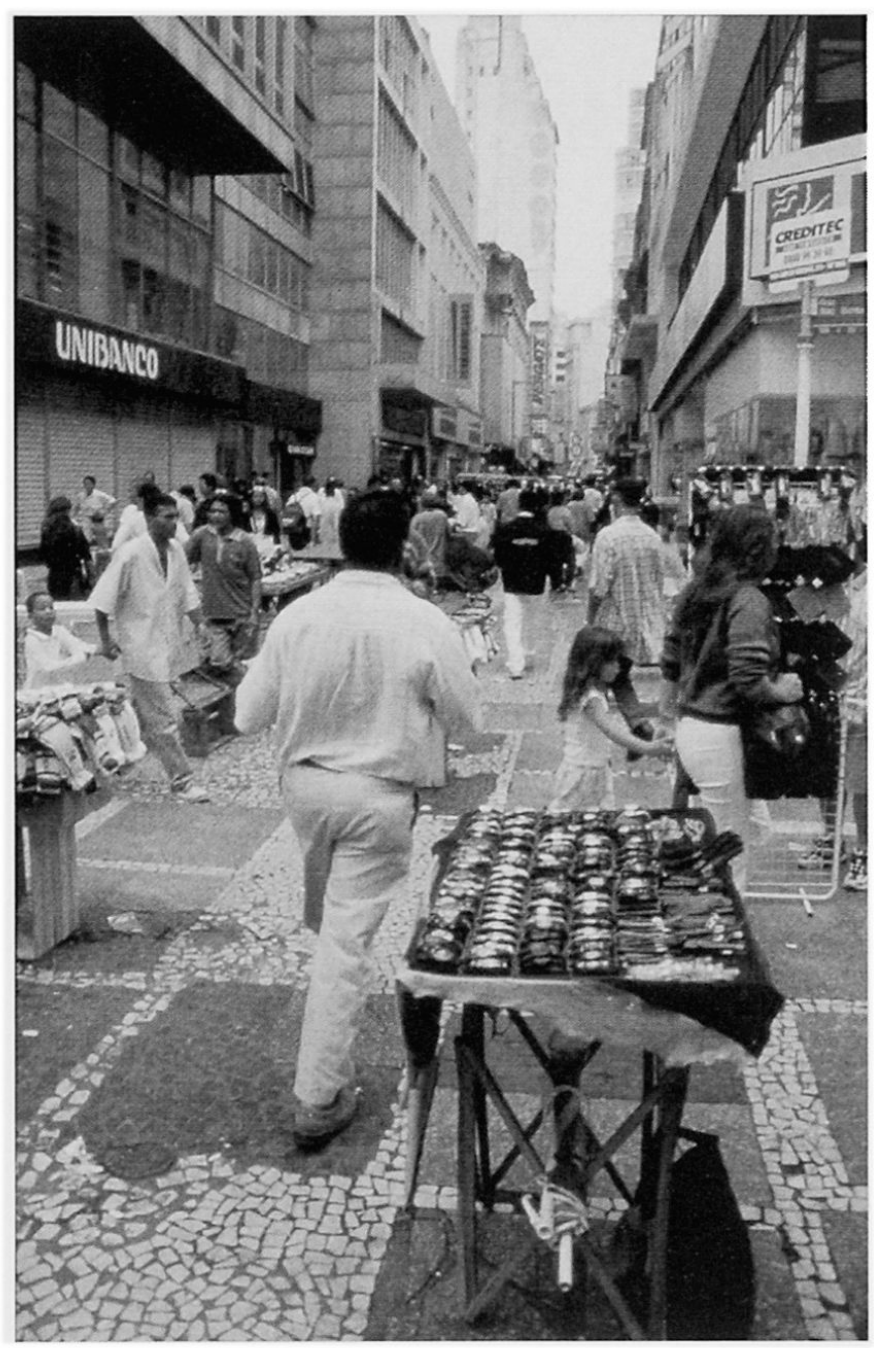

Foto 3: Informeller Strassenhandel im Stadtzentrum von São Paulo

Informal street trade in the city centre of São Paulo Commerce informel de rue au centre de São Paulo Foto: M. Coy 2000
Bereich der hochrangigen Dienstleistungen wird allgemein die zunehmende Besetzung des öffentlichen Raumes sowohl im Centro Velho als auch im Centro Novo durch informelle Tätigkeiten, insbesondere durch den informellen Straßenhandel, als Zeichen des Wandels im Stadtzentrum angeführt (Foto 3). In besonderer Konzentration sind Straßenhändler im öffentlichen Raum einiger traditioneller Einkaufsstraßen zu finden. Nach Angaben von Maricato (1996: 49/50) hat sich in São Paulo zwischen 1982 und 1992 der Anteil der informell Tätigen an der Erwerbsbevölkerung der Stadt ungefähr vervierfacht, was als Indikator für die sich verschärfende sozioökonomische Krisensituation in der Stadt zu werten ist. Dabei steht der informelle Straßenhandel seit Jahren im Mittelpunkt politischer und sozialer Konflikte um die Entwicklung des Stadtzentrums. In verschiedenen großangelegten Polizeiaktionen wurde in den 90er Jahren versucht, den Straßenhandel einzudämmen. Gleichzeitig wurden die Straßenhändler mit der widerrechtlichen Erhebung von «Schutzgeldern» zu Opfern eines des größten Korruptionsskandale in der paulistaner Stadtverwaltung (vgl. FrúGOli JR. 1999). Ein weiterer Aspekt, der in der Diskussion um die Degradierung des Stadtzentrums eine große Rolle spielt, ist die Zunahme von Prostitution und Gewalt (Diebstahl, Raub etc.), wodurch viele Teilräume des Zentrums in der Wahrnehmung der Stadtbevölkerung zu Gefahrenzonen geworden sind.

Insgesamt zeigen die angesprochenen Probleme und Prozesse, daß sich die Funktionen des Stadtzentrums als sozialer Raum während der letzten Jahrzehnte grundlegend gewandelt haben. So haben sich die Bessergestellten als Nutzergruppen weitgehend in die neuen «Wohlstandsenklaven» außerhalb des Stadtzentrums zurückgezogen. Zurück bleiben die sozial Schwächeren und Armen, für die das Stadtzentrum ein nach wie vor wichtiger Aktions- und Überlebensraum ist.

\subsection{Privilegierten-Ghettos - ein neuer Trend sozialräumlicher Fragmentierung}

Deutlicher Beleg der sich vertiefenden sozialräumlichen Segregation sind die abgeschotteten Wohnviertel der Reichen, die sich inzwischen nicht nur in den Metropolen, sondern auch in den Mittelstädten des Landes ausbreiten (vgl. ausführlicher CoY \& PöHLER 2001). Spätestens seit den 80er Jahren werden solche durch Projektentwickler ganzheitlich geplanten Quartiere in immer größerer Zahl und mit großem Erfolg auf dem Immobilienmarkt als neue Produkte angeboten. Diese in Brasilien als condomínios fechados bezeichneten neuen Privilegierten-Viertel orientieren sich eindeutig am nordamerikanischen Vorbild der gated communities (vgl. BLAKELY \& SNYDER 1999), ja übertreffen sie hinsichtlich des Abschottungsgrades noch in vielen Fällen. Ihr Erfolg erklärt sich 
hauptsächlich aus einer begründeten, oftmals auch übersteigerten Angst vor Kriminalität. Die Realisierung einer «privaten Idylle» erscheint angesichts der sich verschärfenden sozialen Konflikte in den Städten nur «unter Seinesgleichen» möglich. Ein immer deutlicherer Trend zur Auto-Segregation ist deshalb die Folge.

So findet sich beispielsweise im nordwestlichen Teil der Metropolitanregion von São Paulo, ungefähr $25 \mathrm{~km}$ von der Kernstadt entfernt auf dem Territorium der Gemeinde Barueri, die Siedlung Alphaville, eine der ältesten und inzwischen eine der größten gated communities Lateinamerikas (vgl. RoMERo 1997). Seit 1975 sind hier insgesamt 15 abgeschlossene Wohngebiete entstanden, in denen sowohl große luxuriös ausgestattete Appartmenthaus-Komplexe als auch Villenviertel hohen Standards angelegt wurden. Für das Jahr 2000 wird die Wohnbevölkerung von Alphaville mit ca. 32.000 angegeben. Die Tagbevölkerung in den Wohngebieten, Einzelhandels-, Büro- und Dienstleistungsstandorten des Projektes soll sich jedoch bereits auf ca. 130.000 belaufen, denn inzwischen existieren in Alphaville über 1.400 Firmen hauptsächlich aus dem Dienstleistungssektor (Firmenverwaltungen, Unternehmen der Computerbranche etc.). Alphaville verfügt über mehrere Shopping Center, zahlreiche Privatschulen, eine Privat-Universität, vielfältige Freizeiteinrichtungen (mehrere Kino-Center,Sportanlagen etc.), über das einzige auf ein privates Siedlungsprojekt beschränkte Kabel-TV Brasiliens sowie über verschiedene eigene Zeitungen, die über das Internet verbreitet werden. Aufgrund der Standorteinheit von Arbeitsstätten, Wohnen, Versorgungs- und Freizeitfunktionen entspricht Alphaville weitgehend dem Muster nordamerikanischer edge cities (vgl. hierzu CALDEIRA 1996: $311 \mathrm{ff}$.).

Nach Untersuchungen eines paulistaner Meinungsforschungsinstitutes sollen ca. $45 \%$ der Haushalte von Alphaville über ein Einkommen zwischen 20 und 80 Mindestlöhnen (der gesetzliche Mindestlohn liegt in Brasilien derzeit bei ca. 100 US-\$) und $35 \%$ der Haushalte sogar von mehr als 80 Mindestlöhnen verfügen. Mehr als $60 \%$ der Haushaltsvorstände haben einen Hochschulabschluß (vgl. Alphanews 87, 2000). Nach derselben Untersuchung haben $64 \%$ der Haushalte in Alphaville gegenüber lediglich $7 \%$ im nationalen Durchschnitt Zugang zum Internet. Dies zeigt eine bewußte globale Orientierung der gated communityBewohner, die sich auf diese Weise im Gegensatz zu ihrer lokalen Auto-Segregation einer global community der Privilegierten zugehörig fühlen.

Die neuen Tendenzen der Wohnstandortwahl der Gutsituierten wirken sich in vielfältiger Weise auf die gesellschaftlichen und (sozial)räumlichen Strukturen aus.
Die gruppenspezifischen alltäglichen Aktionsräume der Privilegierten konzentrieren sich zunehmend auf zugangskontrollierte Enklaven (Wohn-Ghettos, Shopping Center, Gewerbe-Parks) (vgl. FrúGolı JR. 1995). Insofern entsprechen gated communities neuen «exterritorialen Räumen», die sich weitgehend der öffentlichen Steuerung und Kontrolle entziehen. Denn in den meisten Fällen steht öffentliche Regulierung (beispielsweise durch Flächennutzungsplanung oder konkrete Bauvorschriften) eindeutig hinter den Verwertungsinteressen des privaten Kapitals zurück. Dadurch werden die Trennlinien zwischen öffentlichem und privatkapitalistisch kontrolliertem Raum zunehmend unüberbrückbar. Fragmentierung wird auch kleinräumig immer sichtbarer, so daß die brasilianische Stadt heute mehr denn je dem Bild von den «Inseln der Reichen in Ozeanen der Armen» entspricht.

\subsection{Ansätze zu einer Erneuerung der Innenstadt}

Angesichts der in vielen brasilianischen Städten feststellbaren Degradierungstendenzen in den Stadtzentren im Gefolge einer undifferenziert verfolgten Modernisierung erhalten Altstadtsanierung und Innenstadterneuerung als wichtige stadtpolitische Handlungsbereiche in den letzten Jahren einen hohen Stellenwert. Gemeinsames Ziel von Programmen der Sanierung historischer Altstädte und der Innenstadtrevitalisierung ist es, zum einen das lokale historische Erbe (Bausubstanz, öffentliche Räume usw.) zu bewahren und die urbane Attraktivität der Innenstadtbereiche zu steigern und zum anderen die infrastrukturellen Rahmenbedingungen zur Verbesserung von Innenstadtstandorten (sowohl Gewerbe- als auch Wohnfunktion) zu schaffen. In vielen Fällen ist mit einer Revitalisierung der Innenstadt jedoch die Gefahr verbunden, daß sozial schwächere Bewohnergruppen und andere Akteure (insbesondere der informelle Straßenhandel) aus den Innenstadtbereichen verdrängt werden und eine Gentrifizierungstendenz - bewußt oder unbewußt - eingeleitet wird. Ebenso ist als Folge mancher Sanierungsprojekte zu beobachten, daß anstelle eines organischen und vitalen Innenstadtlebens einer eher sterilen «Musealisierung» insbesondere der Altstadtbereiche Vorschub geleistet wird, von der allenfalls der Tourismus profitieren kann (als Beispiel für diese Tendenz wird in Brasilien die Erneuerung des Pelourinho in Salvador angeführt, vgl. Augel 1998).

Auch in der größten Stadt Brasiliens haben Attraktivitätssteigerung und Revitalisierung der Innenstadt angesichts der oben beschriebenen Degradierungstendenzen in den 90er Jahren und bis heute einen zunehmenden Stellenwert erlangt (vgl. SimõES JR. 1994: $37 \mathrm{ff}$.). So hat auch die vor wenigen Monaten angetretene progressive Stadtregierung unter der Leitung der Bürgermeisterin MarTha Suplicy die Revitalisierung 


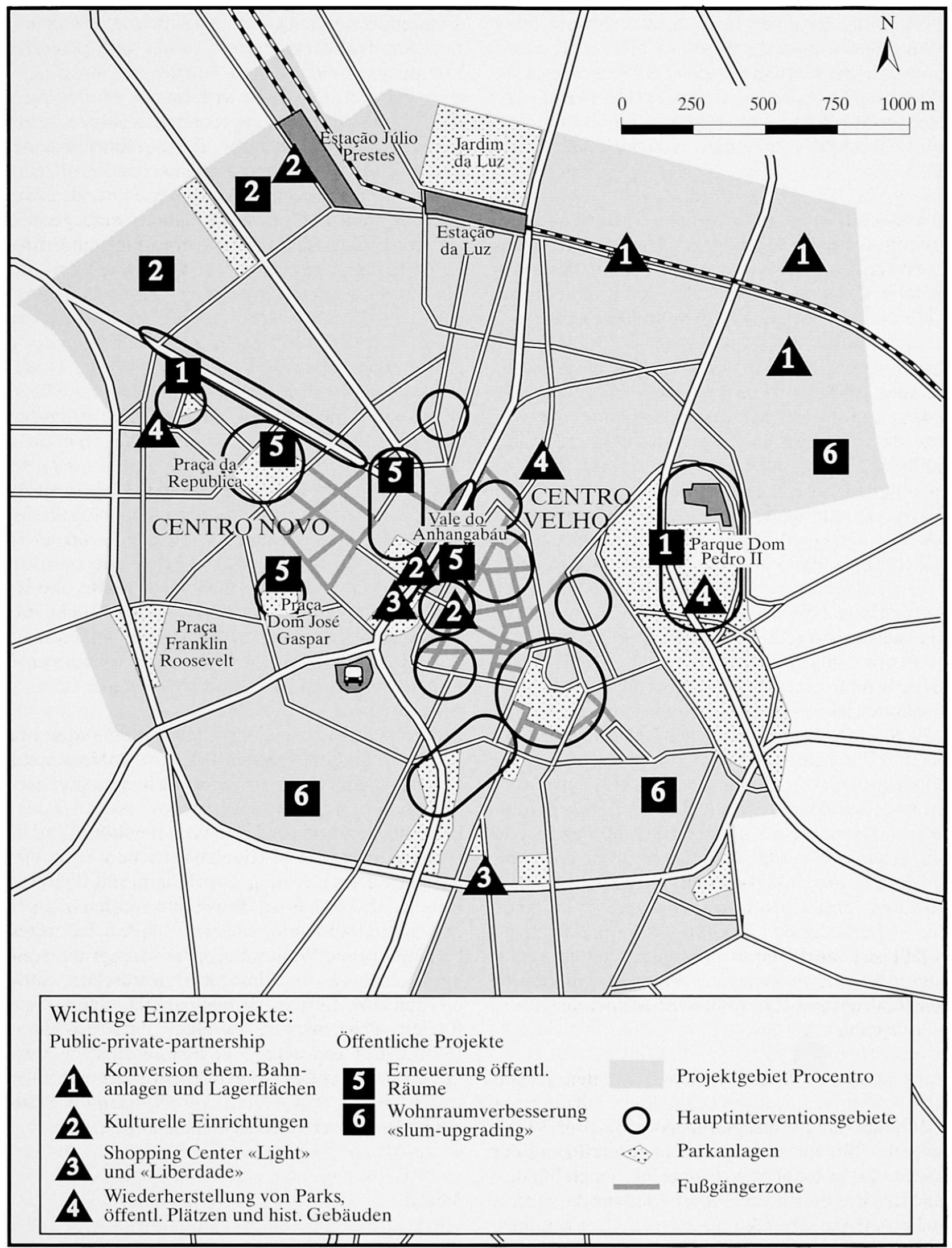

Abb. 6: Aktuelle Projekte der Innenstadterneuerung in São Paulo

Current projects for inner city urban renewal in São Paulo

Projets actuels d'innovation urbaine en centre-ville de São Paulo

Quelle: eigene Zusammenstellung nach Unterlagen PMSP/SEHAB (Prefectura Municipal de São Paulo/ Secretaria de Habitação) 2000 
des paulistaner Stadtzentrums zu einem prioritären Handlungsfeld erklärt. Am Anfang einer Politik der Innenstadterneuerung standen in São Paulo ab Ende der 80er Jahre Maßnahmen zur Verkehrsberuhigung des Zentrums und zur Verbesserung der Qualität des öffentlichen Raumes. So wurden zahlreiche Straßen des Centro Velho und des Centro Novo in Fußgängerzonen umgewandelt, deren Länge sich inzwischen auf ca. $10 \mathrm{~km}$ beläuft. Ein Großprojekt von besonderer Bedeutung war die gelungene Umgestaltung des Vale do Anhangabaú von einer lärmenden Verkehrsader zu einem öffentlichen Raum mit hoher Aufenthaltsqualität. Hinzu kommt die Sanierung und Umnutzung von wichtigen Gebäuden: beispielsweise die Sanierung eines der ältesten Hochhäuser der Stadt, des Edificio Martinelli, in dem heute verschiedene Dezernate der Stadtverwaltung untergebracht sind, die Erneuerung eines der zentralen Bahnhöfe (Estação Júlio Prestes) und seine teilweise Umwandlung in einen riesigen Konzertsaal (Sala São Paulo), die Erhaltung des alten Verwaltungsgebäudes der Elektrizitätsgesellschaft Light und seine Konversion in ein Shopping Center, die Umwandlung der ehemaligen Zentralpost in ein Kulturzentrum und viele andere Vorhaben mehr (vgl. zu den unterschiedlichen Revitalisierungsprojekten Abb. 6). Die Stadtverwaltung ist seit einigen Jahren - allerdings mit wechselndem Erfolg - bemüht, die zahlreichen Einzelprojekte durch ein Rahmenprogramm, das den Namen PROCENTRO führt und an dem zahlreiche Regierungs- und Nichtregierungsinstitutionen beteiligt sind, zu koordinieren und zu unterstützen. Ebenso sollen in einem Spezialprogramm (sogenannte Operação Urbana Centro) mit angepaßten baugesetzlichen Regeln und Anreizsystemen die Rahmenbedingungen für die erhaltende Erneuerung des Zentrums geschaffen werden. Insbesondere die im Zentrum ansässigen Firmen haben großes Interesse an den Revitalisierungsmaßnahmen. Dies läßt sich auch daran erkennen, daß mit zahlreichen public-private-partnerships die Privatinitiative bei der Realisierung der Projekte zunehmende Bedeutung erlangt (vgl. Abb. 6).

Einen besonderen Stellenwert hat bei den Bemühungen um eine Revitalisierung des Stadtzentrums von São Paulo die Nichtregierungsorganisation Viva o Centro, die 1991 unter maßgeblicher Beteiligung der im Centro Velho lokalisierten brasilianischen Niederlassung der Bank of Boston gegründet wurde (vgl. als kritische Analyse der Aktivitäten von Viva o Centro Oliveira 1999). An der relativ ungewöhnlich zusammengesetzten Nichtregierungsorganisation beteiligen sich unterstützend neben der bereits erwähnten Bank zahlreiche weitere Firmen aus dem Finanzsektor sowie verschiedene Interessengruppen und Berufsorganisationen der im Zentrum vertretenen Gewerbe. Auf dieser Grundlage entwickelte sich eine professionell arbeitende Organisation, die den Gedanken der Innenstadt-Revitalisierung sowohl mit planerischer Kompetenz und wissenschaftlichem Anspruch als auch mit öffentlichkeitswirksamem Marketing vertritt. So wurden in den vergangenen Jahren verschiedene Großveranstaltungen durchgeführt, auf denen die unterschiedlichsten Probleme des Zentrums und adäquate Planungsvorhaben diskutiert wurden.Ebenso betreibt Viva o Centro seit Jahren eine geschickte Lobbyarbeit, mit der die Stadtverwaltung mit Erfolg zur Formulierung und zumindest ansatzweisen praktischen Umsetzung einer konkreten Zentrumspolitik gezwungen wurde. Inzwischen unterstützt Viva o Centro in einem speziellen Programm unter dem Namen Ações Locais zahlreiche private Initiativen von Gewerbetreibenden und Bewohnern in den unterschiedlichsten Teilen des Stadtzentrums, deren Ziel es ist, nicht nur auf Mißstände im engeren Umfeld hinzuweisen, sondern auch selbstverantwortlich und selbstverwaltet Sanierungsmaßnahmen durchzuführen (ein recht gelungenes Beispiel ist in diesem Zusammenhang die Sanierung und Revitalisierung der Grandes Galerias am Largo Paissandu). Im Vordergrund steht jedoch insgesamt das Eigeninteresse der beteiligten Wirtschaftskreise, die in der oben beschriebenen Degradierungs- und Verlagerungstendenz auf längere Sicht eine Gefährdung des Standortimages sehen, von dem dann auch die im Zentrum verbliebenen Firmen betroffen wären.

Das Gesamtkonzept der paulistaner Innenstadterneuerung und die meisten Einzelmaßnahmen ähneln auffällig dem, was aus europäischen und nordamerikanischen Städten bekannt ist: Konversion von Brachflächen, Umwandlung von Bahnhöfen und historischen Gebäuden in Kulturhäuser und Einkaufszentren, Wiederherstellung und Möblierung öffentlicher Räume. Die Frage ist, wem diese Maßnahmen letztendlich nützen werden. Zwar werden Betroffenenbeteiligung und Wohnraumverbesserungsmaßnahmen betont, jedoch ist schon jetzt abzusehen, daß die meisten Revitalisierungsmaßnahmen neue «Inseln» für die Privilegierten in einer durch Armut und Informalität geprägten Umgebung schaffen werden. $\mathrm{Ob}$ auch hier wie bei vielen Innenstadtsanierungsvorhaben zusätzlich großflächige gentrification-Prozesse ausgelöst werden, wird sich in Zukunft zeigen.

\section{Fazit}

Die heutige Situation in den brasilianischen Städten im allgemeinen und insbesondere in der Megastadt São Paulo ist nichts anderes als das Abbild einer ungleichen Gesellschaft, die trotz aller makroökonomischen Entwicklungs- und Stabilisierungserfolge der letzten Jahrzehnte nicht in der Lage ist, soziale Konflikte dauerhaft zu lösen und die Lebenssituation des Großteils 
der Bevölkerung nachhaltig zu verbessern. Auch Neoliberalismus und Einbindung des Landes in die Globalisierung haben widersprüchliche Ergebnisse und wirken sich auf die Stadt in zunehmender sozioökonomischer und räumlicher Fragmentierung aus. Zweifellos profitieren auf verschiedenen Maßstabsebenen ganze Regionen, Städte, beziehungsweise Teile der städtischen Wirtschaft und Gesellschaft von aktuellen Standortentscheidungen transnationaler Konzerne oder von der boomhaften Orientierung der Wirtschaft auf den Weltmarkt. Neben diesen «Gewinnern» gibt es jedoch viele «Verlierer», die immer mehr ins Hintertreffen geraten. So wird das gesellschaftliche Konfliktfeld Stadt, wie es am Fallbeispiel São Paulo vorgestellt wurde, von einer Reihe höchst widersprüchlicher Prozesse bestimmt, die zu einem immer stärkeren sozialen und wirtschaftlichen Auseinanderklaffen führen. Die sozialen Gruppen sind hiervon allerdings unterschiedlich betroffen. Die Reichen können vieles durch Geld «abpuffern» und sich so auch in unübersichtlichen Situationen einrichten (vgl. z.B. das gated-communityPhänomen). Die Verwundbarsten sind wie eh und je die ärmeren Bevölkerungsschichten, die einen immer größeren Teil der Stadtbewohner ausmachen.

Diese übergeordneten Bedingungen beeinflussen auch, ob einzelne Städte die neuen Handlungsspielräume, die sich ihnen mit dem Redemokratisierungsproze $\beta$ der letzten Jahre in Brasilien bieten, überhaupt nutzen können. So existieren seit Ende der 80er Jahre in Brasilien Bemühungen um eine Neuformulierung von Stadtpolitik und eine Umorientierung von Stadtplanung im Sinne einer «Stadtreform», die im Wesentlichen dem Versuch entsprechen, das «Recht auf die Stadt» für alle sozialen Gruppen der städtischen Gesellschaft einzufordern und zu realisieren (vgl. hierzu ausführlicher CoY \& ZIRKL 2001). Jedoch muß rückblickend festgestellt werden, daß der Umsetzung bedürfnisorientierter und sozialverträglicherer Maßnahmen zahlreiche Hemmnisse entgegenstanden und nach wie vor entgegenstehen, so daß - von wenigen Ausnahmen abgesehen die Forderung nach einer Stadtreform bis auf den heutigen Tag als uneingelöst gelten muß. Konkreter Handlungsbedarf besteht sowohl auf der Ebene des Stadtmanagements als auch hinsichtlich der Durchführung von konkreten Stadterneuerungsmaßnahmen. Neben den am Beispiel São Paulos erläuterten Maßnahmen zur Revitalisierung der Innenstädte sind dies insbesondere die Verbesserung der Wohnsituation, die Lösung der Verkehrsproblematik sowie das kommunale Umweltmanagement. Viele sinnvolle Ansätze scheitern an der katastrophalen finanziellen Situation der meisten Städte oder auch an nach wie vor bestehenden klientelistischen Politikstrukturen.

An umwelt- und sozialverträglichen Leitbildern zukünftiger Stadtentwicklung und sogar an Konzepten zu ihrer Umsetzung mangelt es in der brasilianischen Diskussion um die Zukunft der Stadt nicht. Immer mehr Planer und Lokalpolitiker haben beispielsweise Begriffe wie Nachhaltigkeit und Partizipation als festen Bestandteil zumindest in ihren Diskurs übernommen. Insbesondere die südbrasilianischen Metropolen Curitiba und Porto Alegre sind auf dem Weg zur Umsetzung dieser Leitbilder bereits ein gutes Stück vorwärts gekommen (vgl. CoY \& ZIRKL 2001). Dabei lassen sich solche positiven Beispiele nicht einfach auf andere Konstellationen, beispielsweise auf die ungleich komplexere Situation in der immer unkontrollierbarer werdenden Megastadt São Paulo, übertragen. Lernen kann man allerdings von diesen best practices, und diese Erkenntnis hat sich heute in vielen brasilianischen Stadtverwaltungen durchgesetzt. Dies gibt Anlaß für einen vorsichtigen Optimismus. Die Kernfrage bleibt allerdings, wie es unter den für die städtischen Handlungsspielräume in vieler Hinsicht ungünstigen Rahmenbedingungen und angepaßt an die jeweiligen lokalen Notwendigkeiten gelingt, substantielle Schritte zur Verbesserung der Lebensbedingungen aller Stadtbewohner zu unternehmen.

\section{Literatur}

Augel, J. (1998): Sustentabilidad de la revitalización. El casco antiguo de Salvador/Bahia, Brasil. - Working Paper $\mathrm{N}^{\circ} 294$, Universität Bielefeld, Forschungsschwerpunkt Entwicklungssoziologie, Bielefeld.

BäHr, J. \& G. Mertins (1995): Die lateinamerikanische Groß-Stadt. Verstädterungsprozesse und Stadtstrukturen. - = Erträge der Forschung 288, Darmstadt: Wissenschaftliche Buchgesellschaft.

BLAKELY, E.J. \& M.G. SNYDER (1999): Fortress America. Gated Communities in the United States. - Washington, Cambrigde/Mass.: Brookings Institution Press.

BoNDUKI, N. (1998): Origens da habitação social no Brasil. Arquitetura Moderna, Lei do Inquilinato e Difusão da Casa Própria. - São Paulo: Ed. Estação Liberdade

Caldeira, T.P.R. (1996): Fortified enclaves: The new urban segregation. - In: Public Culture 8: 303-328.

Coy, M. (1999): Städtischer Strukturwandel und Planung an der brasilianischen Peripherie. Das Beispiel Cuiabá. - In: Trialog. Zeitschrift für das Planen und Bauen in der Dritten Welt 61, 2: 37-43.

Coy, M. \& M. PöHLER (2001): Wohnghettos der Privilegierten und die Fragmentierung der brasilianischen Stadt. Fallbeispiele aus Rio de Janeiro und São Paulo. - In: Matices. Zeitschrift zu Lateinamerika, Spanien und Portugal 8, 29: 8-12.

CoY, M. \& F. ZIRKL (2001): Handlungsfelder und Lösungsansätze nachhaltiger Stadtentwicklung in der Dritten Welt. Beispiele aus Brasilien. - In: Petermanns Geographische Mitteilungen 145, 5: 74-83.

FriedmanN, J. (1995): Ein Jahrzehnt der World City- 
Forschung. - In: Hrtz, H. et al. (Hrsg.): Capitales fatales. Urbanisierung und Politik in den Finanzmetropolen Frankfurt und Zürich. - Zürich: Rotpunktverlag: 22-44. Frúgoli JR., H. (1995): São Paulo: espaços públicos e interação social. - São Paulo: Marco Zero.

FrúGOLI JR., H. (1999): A questão dos camelôs no contexto da revitalização do centro da metrópole de São Paulo. - In: SouzA, M.A.A. DE et al. (Hrsg.): Metrópole e globalização. Conhecendo a cidade de São Paulo. São Paulo: Ed. CEDESP: 151-165.

GUTBERLET, J. (1991): Industrieproduktion und Umweltzerstörung im Wirtschaftsraum Cubatão/São Paulo (Brasilien). - = Tübinger Geographische Studien 106, Tübingen.

KoHLHEPP, G. (1997): São Paulo: größter industrieller Ballungsraum Lateinamerikas. - In: Der Bürger im Staat 47, 2: 137-143.

Kowarick, L. \& C. Ant (1994): Cem anos de promiscuidade: o cortiço na cidade de São Paulo. - In: Kowarick, L. (Hrsg.): As lutas sociais e a cidade. - São Paulo: Ed. Paz e Terra: 73-93.

Maricato, E. (1996): Metrópole na periferia do capitalismo: illegalidade, desigualidade e violência. - São Paulo: Editora Hucitec.

Oliveira, M.A. Aguilar J. de (1999): Associação Viva o Centro: Estratégias, Propostas e Compromissos para São Paulo. - São Paulo, Univ. Mackenzie, Tese de Mestrado. Romero, A.M. (1997): Alphaville: Ilusão do paraíso. São Paulo, Tese de Mestrado, USP/FFLCH.

Santos, M. (1990): Metrópole corporativa fragmentada. O caso de São Paulo. - São Paulo: Nobel.

SANTos, R.C.B. Dos (1994): Rochdale e Alphaville: Formas diferenciadas de apropriação e ocupação da terra na metrópole paulistana. - São Paulo, Tese de doutoramento, USP/FFLCH.

SASSEN, S. (1996): Metropolen des Weltmarkts. Die neue Rolle der Global Cities. - Frankfurt am Main u.a.: Campus-Verlag.

Scholz, F. (2000): Perspektiven des «Südens» im Zeitalter der Globalisierung. - In: Geographische Zeitschrift 88, 1:1-20.

Sımões JR., J. G. (1994): Revitalização de Centros Urbanos. - In: Publicações Pólis 19, São Paulo.

Sомекн, N. (1997): A cidade vertical e o urbanismo modernizador. São Paulo 1920-1939. - São Paulo: Ed. Nobel.

SouzA, M.A.A. DE (1994): A identidade da metrópole. A verticalização em São Paulo. - São Paulo: Editora Hucitec.

VILlaçA, F. (1998): Espaço intra-urbano no Brasil. São Paulo: Ed. Nobel.

\section{Zusammenfassung: São Paulo. Entwicklungstrends einer brasilianischen Megastadt}

Brasilien hat in der zweiten Hälfte des 20. Jahrhunderts einen auf gesellschaftliche und wirtschaft- liche Modernisierung ausgerichteten Entwicklungsweg beschritten. Dies hat einen außerordentlichen Verstädterungsprozeß hervorgerufen. Insbesondere der Ballungsraum São Paulo hat von der ökonomischen Dynamik profitieren können und ist inzwischen zur größten Megastadt Lateinamerikas aufgestiegen. Mit dem rapiden Stadtwachstum sind jedoch tiefgreifende Veränderungen der innerstädtischen Strukturen verbunden. Die folgenden Trends lassen sich herausfiltern: die Zunahme sozialräumlicher Segregation durch die Ausdehnung der Marginalviertel einerseits und abgeschotteter Wohnghettos der Privilegierten andererseits. Damit einher ging eine allmähliche funktionale Degradierung der Innenstadt durch Verdrängung der Wohnbevölkerung sowie eine parallele Randwanderung von Zentrumsfunktionen an neue Entwicklungsachsen bzw. in die zahlreichen neuen Shopping Center. Vor diesem Hintergrund werden die aktuellen Bemühungen von Stadtverwaltung und NichtRegierungsorganisationen um eine Revitalisierung des Stadtzentrums untersucht.

\section{Summary: São Paulo. Development trends of a Brazilian megacity}

During the second half of the 20th century, development in Brazil was focussed on the modernisation of its society and economy. This led to an exceptional process of urbanisation. As a result of the resulting economic dynamic generated, the conurbation of São Paulo was able to profit most, growing to become the largest megacity in Latin America. The consequence of rapid urban growth was a profound change of inner city structures. The following trends may be defined: increasing socio-spatial segregation due to the expansion of marginalized areas and the upswing of gatedcommunities for the privileged. Concomitant to these trends were the gradual functional degradation of the inner city due to the displacement of the residential population and the dislocation of central functions along development axes, respectively to numerous new shopping centres on the outskirts of the city. The current efforts of the municipality and non-governmental organisations to regenerate the urban centre are looked at in the context of the above.

\section{Résumé: São Paulo: tendances de l'évolution d'une mégapole brésilienne}

Le Brésil s'est engagé durant la seconde moitié du $\mathrm{XX}^{\mathrm{c}}$ siècle sur une voie de développement résolument orientée vers la modernisation sociétale et économique. Cette transformation a suscité un extraordinaire processus d'urbanisation. L'espace de densification urbaine extrême de São Paulo a bénéficié tout particulièrement de cette dynamique économique, à tel point qu'il est parvenu entretemps à se positionner en tête des mégapoles latino-américaines. De profondes modifications des structures intraurbaines sont cepen- 
dant liées à cette rapide croissance urbaine. Il est possible d'esquisser dès à présent les tendances suivantes: le renforcement de la ségrégation socio-spatiale par l'extension des quartiers marginaux d'une part, par la ghettoisation des catégories privilégiées de la population d'autre part. Cette évolution s'est accompagnée d'une dégradation fonctionnelle progressive du centreville par l'élimination de la population résidente, ainsi que par un mouvement corrélatif de migration de certaines fonctions centrales vers la périphérie urbaine, le long de nouveaux axes de développement ou en direction des nombreux nouveaux centres commerciaux créés en espace périurbain. Face à cet arrièreplan, le présent article analyse les efforts actuels de l'administration urbaine et des organisations non-gouvernementales en faveur d'une revitalisation du centre-ville.

PD Dr. Martin Coy, Geographisches Institut, Universität Tübingen, Hölderlinstraße 12, D-72074 Tübingen.

e-mail:martin.coy@uni-tuebingen.de

\section{Manuskripteingang/received/manuscrit entré le} 13.9.2001

Annahme zum Druck/accepted for publication/accepté pour l'impression: 14.12.2001 